\title{
Helical Force Effects on the Onset of Küppers-Lortz Instability with Free-Free Boundaries
}

\author{
Marcelline Essoun ${ }^{1,2} \&$ Jean B. Chabi Orou ${ }^{1,2}$ \\ ${ }^{1}$ Institut de Mathématiques et de Sciences Physiques (IMSP), 01 BP 613 Porto-Novo, Bénin \\ ${ }^{2}$ The Abdus Salam International Centre for Theoretical Physics, Trieste, Italy \\ Correspondence: Jean B. Chabi Orou, Institut de Mathématiques et de Sciences Physiques (IMSP), 01 BP 613 \\ Porto-Novo, Bénin. Email: jean.chabi@imsp-uac.org
}

Received: June 6, 2012 Accepted: June 24, 2012 Online Published: July 16, 2012

doi:10.5539/apr.v4n3p61 URL: http://dx.doi.org/10.5539/apr.v4n3p61

\begin{abstract}
The rotating Rayleigh-Bénard convection in the presence of helical force is modeled by a four mode Lorenz model. This model is extended to study how this force affects the onset of Küppers-Lortz (KL) instability. We observed that when $S<3 \pi$, the critical Taylor number and the critical angle for the onset of KL instability decrease as helical force intensity $S$ increases. This influence of helical force is similar to that obtained in rotating fluid layer under periodic modulation of the rotation rate (Bhattacharjee, 1990). In the range $3 \leq S$ $\leq 14.9246$, the system exhibits the reentrant behavior of rolls demonstrating the constructive and destructive role of rotation in the KL instability apparition. In this case, we observed that the application of this force allows the KL instability for small values of Taylor number. In addition, it has been found that there exists a threshold (14.9246) in the magnitude of the helical force that allows suppressing the KL instability in the system for any value of Taylor number.
\end{abstract}

Keywords: rotating Rayleigh-Bénard convection, helical force, Lorenz model, Küppers-Lortz instability

\section{Introduction}

Rayleigh-Bénard convection in a plane layer heated from below and rotating about a vertical axis, has been the objects of special attention motivated by both astrophysical and geophysical applications and by the existence of additional instabilities occuring in this system. Moreover, rotating convection generates some interesting dynamics with a much more complicated structure than in the non-rotating case. In the nonlinear regime of rotating Rayleigh-Bénard convection, the most interesting phenomenon is the occurence of KL instability. Küppers and Lortz (1969) reported that for an infinite Prandtl number in a rotating Rayleigh-Bénard system with free-free boundary conditions, at the onset of stationary convection, the roll solution was unstable to perturbations by rolls with different axes when the Taylor number (which measures the rotation rate) exceeds the critical value 2285 and the angle close to $58^{\circ}$ These new sets of rolls are similarly unstable. This process continues resulting in complex dynamics. This leads to the onset of a complicated spatio-temporal complex state and possible chaotic behavior at the onset when the Taylor number exceeds a certain threshold. This instability was observed by Busse and Heikes (1980). Many experiment and theoretical (Niemela \& Donnelly, 1986; Liu \& Ahlers, 1997; Clune \& Knobloch, 1993; Murali et al., 1994; Ponty et al., 1997; Cox \& Mattews, 2000) research papers exist on KL instability.

The Lorenz model was first obtained by E. N. Lorenz (1963) when he modeled the convection in the atmosphere. This model represents the Rayleigh-Bénard convection for both parallel and circular plates (Sparrow, 1982; Tritton, 1988). This apparently simple nonlinear model has generated thousands of research papers and it continues generating more due to the fact that the system is not completely understood. Some properties of the Lorenz equations are summarized in (Sparrow, 1982). Bhattacharjee (Bhattacharjee \& Mckane, 1988) and Stein (Stein, 1989) have modeled the rotating Rayleigh-Bénard convection by a four-mode Lorenz model. J. K. Bhattacharjee and A. J. Mckane (1988) have extented this model for studying the KL instability.

The generating properties of small-scale helical turbulence leading to the large-scale structure formation were first discovered in magnetohydrodynamics (Steenbeck et al., 1966). This phenomenon is known as the alpha-effect. The realizability of the hydrodynamic $\alpha$ - effect, the analogous phenomenon in 
non-electroconductive media, has been theoretically justified for compressible (Moiseev et al., 1983) and, later, incompressible (Moiseev et al., 1988) fluids. Publications on the hydrodynamic alpha-effect were examined in detail in review (Rutkevich, 1993; Kurgansk, 1998; Levina et al., 2000). In (Levina et al., 2000), the authors have proposed the special force also called helical force that has the structure of the alpha term, which provides excitation of large-scale instability within the framework of the model of the hydrodynamic alpha-effect in a convective system. This force simulates the influence of small-scale helical turbulence which is generated in a rotating fluid with internal heat sources (Rutkevich, 1993).

The linear stability analysis of the rotating Rayleigh-Bénard system in the presence of this force has been performed in the case of free-free boundary conditions (Essoun \& Chabi Orou, 2010). It was found that this helical force has no monotonic effects on the onset of convection for all values of Taylor number but decreases the threshold of the corresponding critical wave number for any value of Taylor number.

In this work, we have considered rotating Rayleigh-Bénard problem in the presence of helical force. In the next section, we obtain the Lorenz-type model for this system that is extended within the framework to study how this force affects the onset of KL instability. In section 3, we summarize our results.

\section{Derivation of the Model}

We consider a mathematical model described in (Levina, 2006) including the nonlinear Boussinesq equations, in which the momentum equation includes the helical and Coriolis forces. The dimensionless form is written as:

$$
\begin{gathered}
\frac{\partial \overrightarrow{\mathrm{v}}}{\partial \mathrm{t}}+\frac{1}{\operatorname{Pr}} \overrightarrow{\mathrm{v}} \cdot \nabla \overrightarrow{\mathrm{v}}=-\nabla \mathrm{p}+\nabla^{2} \overrightarrow{\mathrm{v}}+\operatorname{Ra} \mathrm{T} \overrightarrow{\mathrm{e}}-\mathrm{Ta}^{1 / 2}(\overrightarrow{\mathrm{e}} \times \overrightarrow{\mathrm{v}})+\mathrm{S} \overrightarrow{\mathrm{f}}, \\
\operatorname{Pr} \frac{\partial \mathrm{T}}{\partial \mathrm{t}}+\overrightarrow{\mathrm{v}} \cdot \nabla \mathrm{T}=\nabla^{2} \mathrm{~T}, \\
\nabla \cdot \overrightarrow{\mathrm{v}}=0, \\
\overrightarrow{\mathrm{f}}=\overrightarrow{\mathrm{e}}(\operatorname{curl}(\overrightarrow{\mathrm{v}}))_{\mathrm{z}}-\frac{\partial(\overrightarrow{\mathrm{e}} \times \overrightarrow{\mathrm{v}})}{\partial \mathrm{z}}, \quad \overrightarrow{\mathrm{e}}=(0,0,1), \\
\mathrm{Ra}=\frac{\beta g\left(\mathrm{~T}_{1}-\mathrm{T}_{2}\right) \mathrm{h}^{3}}{v \kappa}, \quad \operatorname{Pr}=\frac{v}{\kappa}, \quad \mathrm{Ta}=\frac{4 \Omega^{2} \mathrm{~h}^{4}}{v^{2}},
\end{gathered}
$$

Where $\vec{v}$ is velocity, $\mathrm{p}$ is pressure, $T$ is temperature, $\vec{f}$ is helical force, $\overrightarrow{\mathrm{e}}$ is the unit vector along the vertical axis; Ra, Pr and Ta are respectively dimensionless Rayleigh, Prandtl and Taylor numbers; and $\mathrm{S}$ is a dimensionless parameter that characterizes the intensity of force $\vec{f}$. The height of the layer $\mathrm{h}$ and characteristic temperature difference between the boundaries of the layer $\left(T_{1}-T_{2}\right)$, as well as values $\frac{h^{2}}{v}, \frac{\kappa}{v}$ and $\frac{\rho_{0 v \kappa}}{h^{2}}$ were taken as units of measure of length, temperature, time, velocity and pressure respectively.

Equations (1) in the absence of the Coriolis force were scrutinized in (Levina \& Burylov, 2006). The special case $S=0$ corresponds to that in which the helical force is absent, and system (1) is reduced to the equations describing the Rayleigh-Bénard convection with rotation when the centrifugal force is neglected.

The analysis of hydrodynamic equations describing the system requires three independent variables. These are the $z$-component of the velocity field $\mathrm{w}$, the $z$-component of vorticity $\zeta$ and $\theta$ the fluctuations of the temperature.

The governing equations (1) in terms of $\mathrm{w}, \zeta$ and $\theta$ become:

$$
\begin{gathered}
\frac{\partial}{\partial \mathrm{t}}\left(\nabla^{2} \mathrm{w}\right)-\frac{1}{\operatorname{Pr}} \overrightarrow{\mathrm{e}} \cdot[\nabla \times\{(\overrightarrow{\mathrm{v}} \cdot \nabla) \vec{\omega}-(\vec{\omega} \cdot \nabla) \overrightarrow{\mathrm{v}}\}]=\operatorname{Ra} \nabla_{\mathrm{h}}^{2} \theta+\nabla^{4} \mathrm{w}-\mathrm{Ta}^{1 / 2} \mathrm{D} \zeta+S\left(\nabla_{\mathrm{h}}^{2} \zeta-\mathrm{D}^{2} \zeta\right), \\
\frac{\partial \zeta}{\partial \mathrm{t}}+\frac{1}{\operatorname{Pr}}[(\overrightarrow{\mathrm{v}} \cdot \nabla) \zeta-(\vec{\omega} \cdot \nabla) \mathrm{w}]=\nabla^{2} \mathrm{w}+\mathrm{Ta}^{1 / 2} \mathrm{D} \zeta+\mathrm{SD}^{2} \mathrm{w} \\
\operatorname{Pr} \frac{\partial \theta}{\partial \mathrm{t}}+(\overrightarrow{\mathrm{v}} \cdot \nabla) \theta=\mathrm{w}+\nabla^{2} \theta
\end{gathered}
$$

where

$$
\mathrm{D}=\frac{\mathrm{d}}{\mathrm{dz}}, \quad \nabla_{\mathrm{h}}^{2}=\frac{\partial^{2}}{\partial \mathrm{x}^{2}}+\frac{\partial^{2}}{\partial \mathrm{y}^{2}} \quad \text { and } \quad \nabla^{2}=\nabla_{\mathrm{h}}^{2}+\mathrm{D}^{2}
$$


In the absence of helical force $(\mathrm{S}=0)$, Bhattacharjee and Mckane (Bhattacharjee \& Mckane, 1988) have dropped the nonlinear terms in the equations of $\mathrm{w}$ and $\zeta$ to model the system by the four-mode Lorenz equations. They supposed that the use of free-free boundary conditions does not lead to the contributions from these terms in the Lorenz model. Stein (Stein, 1989) considered these nonlinear terms and obtained the same four-mode Lorenz equations. Then, we use the same procedure as Bhattacharjee and Mckane to model our system by Lorenz-type model.

Neglecting the nonlinear terms in the equations of $w$ and $\zeta$, system (2) becomes:

$$
\begin{gathered}
\frac{\partial}{\partial t}\left(\nabla^{2} w\right)=R a \nabla_{h}^{2} \theta+\nabla^{4} w-T a^{1 / 2} D \zeta+S\left(\nabla_{h}^{2} \zeta-D^{2} \zeta\right), \\
\frac{\partial \zeta}{\partial t}=\nabla^{2} w+T a^{1 / 2} D \zeta+S D^{2} w \\
\operatorname{Pr} \frac{\partial \theta}{\partial t}+(\vec{v} \cdot \nabla) \theta=w+\nabla^{2} \theta .
\end{gathered}
$$

The linear stability analysis of system (3) shows that the stationary convection occurs at (Essoun \& Chabi Orou, 2010):

$$
R a_{s c}=\frac{\left(\pi^{2}+k_{s c}{ }^{2}\right)^{3}+\pi^{2}\left(T a-S^{2} \pi^{2}+S^{2} k_{s c}^{2}\right)}{k_{s c}^{2}}
$$

where $\mathrm{k}_{\mathrm{sc}}$ is the positive root of the following equation:

$$
2\left(\frac{k_{s c}}{\pi}\right)^{6}+3\left(\frac{k_{s c}}{\pi}\right)^{4}=1+\frac{T a-S^{2} \pi^{2}}{\pi^{4}} .
$$

We assume that the roll axis is taken to be the $y$ axis. The Lorenz model for the two dimensional rolls can be obtained by introducing the following forms for the different fields (Bhattacharjee \& Mckane, 1988):

$$
\begin{gathered}
w=a(t) \cos \left(k_{s c} x\right) \sin \pi z \\
\zeta=f(t) \cos \left(k_{s c} x\right) \cos \pi z \\
\theta=b(t) \cos \left(k_{s c} x\right) \sin \pi z+c(t) \sin 2 \pi z
\end{gathered}
$$

Inserting system(6) into model (3), we get:

$$
\begin{gathered}
\dot{X}=\sigma(-X+Y+d F) \\
\dot{Y}=r X-Y-X Z \\
\dot{\mathrm{Z}}=-\mathrm{bZ}+\mathrm{XY} \\
\dot{\mathrm{F}}=-\sigma(\mathrm{d} \mathrm{X}+\mathrm{F})
\end{gathered}
$$

where

$$
r=\frac{R a k_{s c}{ }^{2}}{\left(\pi^{2}+{k_{s c}}^{2}\right)^{3}}, \quad b=\frac{4 \pi^{2}}{\pi^{2}+k_{s c}{ }^{2}}, \quad d=\frac{\pi T a^{1 / 2}}{\left(\pi^{2}+k_{s c}\right)^{3 / 2}}, \quad \sigma=\operatorname{Pr},
$$

$\mathrm{X}, \mathrm{Y}, \mathrm{Z}$ and $\mathrm{F}$ are the dimensionless form of $\mathrm{a}, \mathrm{b}, \mathrm{c}$ and $\mathrm{f}$ respectively.

This system has three fixed points: the conduction state $\mathrm{O}(0,0,0,0)$ and the stationary finite amplitude solutions $C^{ \pm}\left(X_{0}^{ \pm}, Y_{0}^{ \pm}, Z_{0}, F_{0}^{ \pm}\right)$, where $X_{0}^{ \pm}= \pm \sqrt{b\left(\frac{r}{1+d^{2}}\right)}, Y_{0}^{ \pm}=\left(1+d^{2}\right) X_{0}^{ \pm}, Z_{0}=r-\left(1+d^{2}\right)$ and $F_{0}^{ \pm}=-d X_{0}^{ \pm}$.

Clearly, the conduction state is a fixed point for all parameter values but exist $\mathrm{C}^{ \pm}$only when $r>1+d^{2}$.

The above model will be extended to include the KL instability and we will address how the helical force affects the onset of KL instability. We assume that the basic rolls (axis along the $y$ direction) can be perturbed by a set of rolls whose axis is not along the $y$ axis and the wave vector makes an angle $\psi$ with the $x$ axis. Then, the wave vector $\overrightarrow{\mathrm{k}}$ of these disturbance rolls would have the components $k_{1}=k_{s c} \cos \psi$ and $k_{2}=k_{s c} \sin \psi$.

We introduce the following expansions that include the $y$ dependence in the fields (Bhattacharjee \& Mckane, 1988):

$$
w=a(t) \cos \left(k_{s c} x\right) \sin \pi z+a_{1}(t) \cos \left(k_{1} x+k_{2} y\right) \sin \pi z
$$




$$
\begin{gathered}
\zeta=\mathrm{f}(\mathrm{t}) \cos \left(\mathrm{k}_{\mathrm{sc}} \mathrm{x}\right) \cos \pi \mathrm{z}+\mathrm{f}_{1}(\mathrm{t}) \cos \left(\mathrm{k}_{1} \mathrm{x}+\mathrm{k}_{2} \mathrm{y}\right) \cos \pi \mathrm{z} \\
\theta=b(t) \cos \left(k_{s c} x\right) \sin \pi z+c(t) \sin 2 \pi z+b_{1}(t) \cos \left(k_{1} x k_{2} y\right) \sin \pi z+\left[c _ { 1 } ( t ) \operatorname { c o s } \left(k_{1} x+k_{s c} x+\right.\right. \\
\left.\left.k_{2} y\right)+c_{2}(t) \cos \left(k_{1} x-k_{s c} x+k_{2} y\right)\right] \sin 2 \pi z
\end{gathered}
$$

Inserting these expansions into equations (3), we get the correspondent Lorenz-type model:

$$
\begin{gathered}
\dot{X}=\sigma(-X+Y+d F) \\
\dot{Y}=r X-Y-X Z-X_{1} Z_{1} \frac{1-\cos \psi}{4}-X_{1} Z_{2} \frac{1+\cos \psi}{4}-F_{1} Z_{1} q \frac{\sin \psi}{4}+F_{1} Z_{2} q \frac{\sin \psi}{4} \\
\dot{Z}=-b Z+X Y+X_{1} Y_{1} \\
\dot{F}=-\sigma(d X+F) \\
\dot{X}_{1}=\sigma\left(-X_{1}+Y_{1}+\mathrm{dF}_{1}\right) \\
\dot{Y}_{1}=r X_{1}-Y_{1}-X_{1} Z-X Z_{1} \frac{1-\cos \psi}{4}-X Z_{2} \frac{1+\cos \psi}{4}+F Z_{1} q \frac{\sin \psi}{4}-F Z_{2} q \frac{\sin \psi}{4} \\
\dot{Z}_{1}=\frac{1-\cos \psi}{2}\left(Y X_{1}+X Y_{1}\right)-b_{+} Z_{1}+q \frac{\sin \psi}{2}\left(F_{1} Y-F Y_{1}\right) \\
\dot{Z}_{2}=\frac{1+\cos \psi}{2}\left(Y X_{1}+X Y_{1}\right)-b_{-} Z_{2}+q \frac{\sin \psi}{2}\left(F Y_{1}-F_{1} Y\right) \\
\dot{F}_{1}=-\sigma\left(d X_{1}+F_{1}\right)
\end{gathered}
$$

where

$$
\begin{gathered}
q=\frac{\left(\pi^{2}+\mathrm{ksc}^{2}\right)^{1 / 2}}{\pi} \\
\mathrm{b}_{ \pm}=\frac{4 \pi^{2}+2 \mathrm{k}_{\mathrm{sc}}{ }^{2}(1 \pm \cos \psi)}{\pi^{2}+\mathrm{k}_{\mathrm{sc}}{ }^{2}},
\end{gathered}
$$

$X_{1}, Y_{1}, Z_{1}, Z_{2}$ and $F_{1}$ are the dimensionless versions of $a_{1}, b_{1}, c_{1}, c_{2}$ and $f_{1}$ respectively. Thus, a nine-mode system (9) is going to describe how the helical force affects the onset of KL instability.

\section{Results and Discussion}

The rolls along $y$ axis are described by:

$$
\begin{gathered}
X_{0}^{2}=b\left(\frac{r}{1+d^{2}}-1\right) \\
Y_{0}=\left(1+d^{2}\right) X_{0} \\
Z_{0}=r-\left(1+d^{2}\right) \\
F_{0}=-d X_{0}
\end{gathered}
$$

These rolls are perturbed by those describing by $\mathrm{X}_{1}, \mathrm{Y}_{1}, \mathrm{Z}_{1}, \mathrm{Z}_{2}$ and $\mathrm{F}_{1}\left(\mathrm{X}_{1}, \mathrm{Y}_{1}, \mathrm{Z}_{1}, \mathrm{Z}_{2}\right.$ and $\mathrm{F}_{1}$ are perturbations rolls). The linear stability analysis of the rolls along y axis against these perturbations will be studied by using a linearized five mode system:

$$
\begin{gathered}
\dot{X}_{1}=\sigma\left(-X_{1}+Y_{1}+d F_{1}\right) \\
\dot{Y}_{1}=r X_{1}-Y_{1}-X_{1} Z-X Z_{1} \frac{1-\cos \psi}{4}-X Z_{2} \frac{1+\cos \psi}{4}+F Z_{1} q \frac{\sin \psi}{4}-F Z_{2} q \frac{\sin \psi}{4} \\
\dot{Z}_{1}=\frac{1-\cos \psi}{2}\left(Y X_{1}+X Y_{1}\right)-b_{+} Z_{1}+q \frac{\sin \psi}{2}\left(F_{1} Y-F Y_{1}\right)
\end{gathered}
$$




$$
\begin{gathered}
\dot{Z}_{2}=\frac{1+\cos \psi}{2}\left(Y X_{1}+X Y_{1}\right)-b_{-} Z_{2}+q \frac{\sin \psi}{2}\left(F Y_{1}-F_{1} Y\right) \\
\dot{F}_{1}=-\sigma\left(d X_{1}+F_{1}\right)
\end{gathered}
$$

We introduce the perturbations of the form $\mathrm{e}^{\lambda \mathrm{t}}$. We find that $\lambda=0$ if:

$$
T a^{1 / 2} \sin \psi=\left(\pi^{2}+k_{s c}{ }^{2}\right) \frac{b_{+}(1+\cos \psi)^{2}+b_{-}(1-\cos \psi)^{2}}{b_{+}(1+\cos \psi)-b_{-}(1-\cos \psi)}
$$

Inserting equation (10) into equation (13), we get:

$$
(x+2) \tan ^{2} \psi-2 \Gamma \tan \psi+4(x+1)=0,
$$

where

$$
\begin{gathered}
x=\frac{k_{s c}^{2}}{\pi^{2}} \\
\Gamma=\frac{T a^{1 / 2}}{\pi^{2}}
\end{gathered}
$$

Solving equation (14) in terms of $\Gamma$ and $x$, we obtain:

$$
\tan \psi=\frac{\Gamma \pm \sqrt{\Gamma^{2}-4(x+1)(x+2)}}{x+2}
$$

The minimum value of Ta will be obtained for:

$$
\mathrm{Ta}=4(\mathrm{x}+1)(\mathrm{x}+2) \pi^{4}
$$

Combining equations (5) and (17), we have:

$$
2 x^{3}-x^{2}-12 x-9+\frac{s^{2}}{\pi^{2}}=0
$$

The critical value of Taylor number and the associated critical angle for the onset of KL instability are given by:

$$
\begin{gathered}
\mathrm{Ta}_{\mathrm{c}}=4(\mathrm{x}+1)(\mathrm{x}+2) \pi^{4} \\
\psi_{c}=\tan ^{-1}\left(2 \sqrt{\frac{x+1}{x+2}}\right)
\end{gathered}
$$

where $x$ is the positive roots of equation (18).

For $S=0$, the critical Taylor number is $T a_{c}=779.728$ and the associated critical angle is $\psi_{\mathrm{c}}=60.794^{\circ}$. Our values match those obtained by Bhattacharjee and Mckane (1988). This value of $\mathrm{Ta}_{c}$ is different from that obtained by Küppers and Lortz because of the approximations. In figures 1(a) and 1(b), we plot the critical Taylor number $\mathrm{Ta}_{\mathrm{c}}$ and the critical angle $\psi_{\mathrm{c}}$ for the onset of KL instability both as function of the helical force intensity S. These curves define the boundary between the stable roll and the KL instability domains. We observe that this model force does not have the uniform effect on the onset of KL instability. There exists two critical values of $S, S_{c r 1}=3 \pi$ and $S_{c r 2} \cong 14.9246$.

For $\mathrm{S}<3 \pi$, equation (18) has one positive root that implies one critical Taylor number and one critical angle for the onset of KL instability. We observe that the threshold values of Taylor number $\mathrm{Ta}_{\mathrm{c}}$ and angle $\psi_{c}$ decrease as $S$ increases (column I). This influence of helical force on the onset of KL instability is similar to that observed by J. K. Bhattacharjee in rotating fluid layer under periodic modulation of the rotation rate (Bhattacharjee, 1990).

For $3 \pi \leq S \leq 14.0246$, equation (18) has two positive different roots that correspond to the two different critical values of Taylor number and associated angle. As one can see from figure 1, by increasing the Taylor number, the system exhibits KL unstable rolls $\leftrightarrow$ stable rolls transitions in the range $3 \pi \leq S \leq 14.0246$. This reentrant behavior of the rolls i.e. as Ta is increased, the $\mathrm{Kl}$ unstable rolls (unstable after Küppers and Lortz view) can become stable and then lose stability, demonstrates the constructive and destructive role of rotation. We also observe that in this reentrant domain (column II), the system allows the K1 instability for small values of 
Taylor number.

For S > 14.9246, equation (18) has no positive root. Then we deduce that the Kl instability can be suppressed by helical force when its intensity $S$ exceeds the critical value 14.9246 (column III).

Let us note $\Delta \mathrm{Ta}_{\mathrm{c}}=\mathrm{Ta}_{\mathrm{c}}(\mathrm{S}=0)-\mathrm{Ta}_{\mathrm{c}}(\mathrm{S} \neq 0)$ and $\Delta \psi_{\mathrm{c}}=\psi_{\mathrm{c}}(\mathrm{S}=0)-\psi_{\mathrm{c}}(\mathrm{S} \neq 0)$, the shift in the value of the critical Taylor number and critical angle for the onset of $\mathrm{KL}$ instability when $S<3 \pi ; \delta \mathrm{Ta}_{\mathrm{c}}=\frac{\Delta \mathrm{Ta}_{\mathrm{c}} \times 100}{\mathrm{Ta}_{\mathrm{c}}(\mathrm{S}=0)}$ and $\delta \psi_{c}=\frac{\Delta \psi_{c} \times 100}{\psi_{c}(S=0)}$ the decreasing percentage of the critical Taylor number and of the corresponding critical angle respectively.
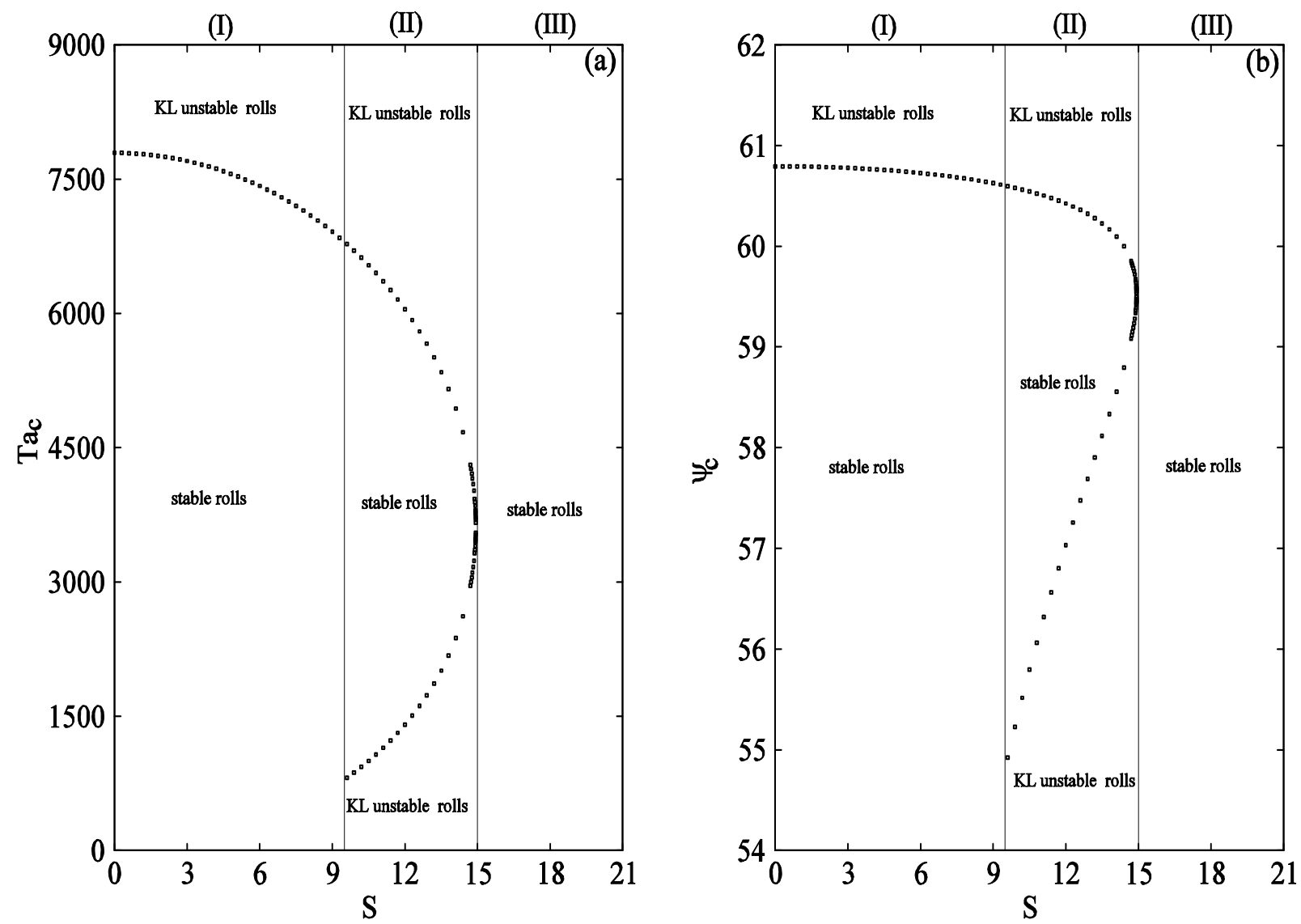

Figure 1. (a) Critical Taylor number $\mathrm{Ta}_{c}$ and (b) associated critical angle $\psi_{\mathrm{c}}$ (in degrees) for the onset of KL instability as function of intensity $\mathrm{S}$ of helical force

In Figure 2, we plot the shift in the value of critical Taylor number and of critical angle for the onset of KL instability as a function of helical force intensity. We observe that $\Delta \mathrm{Ta}_{\mathrm{c}}$ and $\Delta \psi_{\mathrm{c}}$ increase as $\mathrm{S}$ increases. Table below shows the decreasing percentage of the critical Taylor number and of critical angle as a function of helical force intensity $\mathrm{S}$. We observe that the decreasing percentage increases as $\mathrm{S}$ increases. 

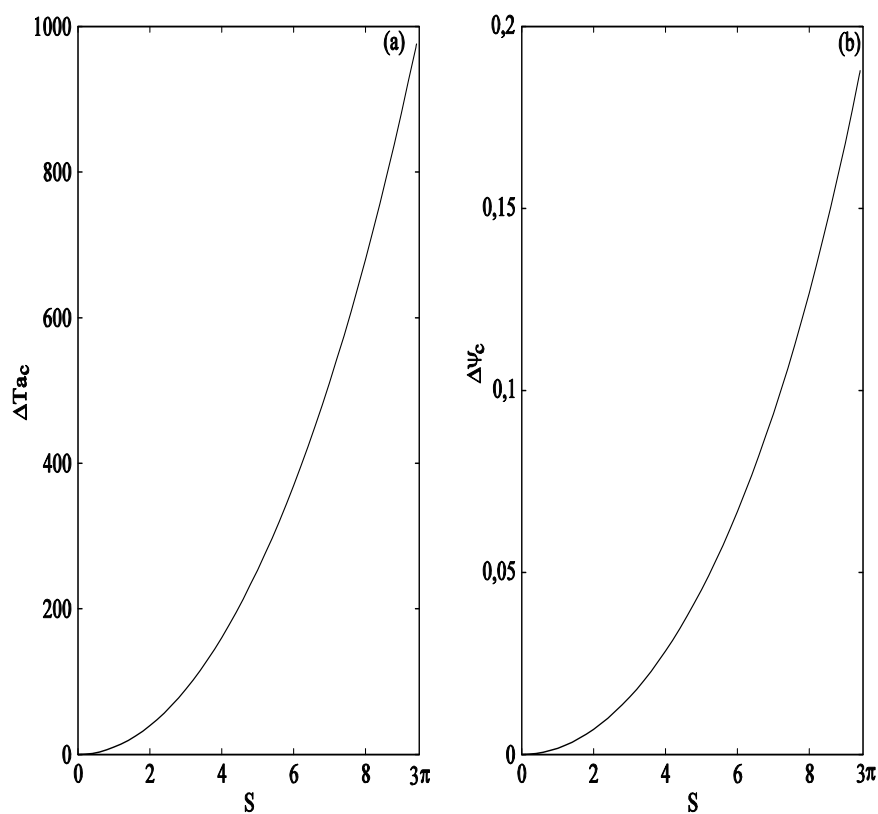

Figure 2. The shift in the value of: (a) critical Taylor number and (b) critical angle as a function of helical force intensity $S$

Table 1. Decreasing percentage of critical Taylor number $\delta T a_{\mathrm{c}}$ and of corresponding critical angle $\delta \psi_{\mathrm{c}}$ versus helical force intensity $S$

\begin{tabular}{ccc}
\hline $\mathrm{S}$ & $\delta \mathrm{Ta}_{\mathrm{c}}$ & $\delta \psi_{\mathrm{c}}$ \\
\hline 0.000 & 0.000 & 0.000 \\
0.100 & 001 & 0.000 \\
0.500 & 0.032 & 0.000 \\
1.000 & 0.127 & 0.003 \\
1.500 & 0.286 & 0.006 \\
2.000 & 2.000 & 0.011 \\
2.500 & 0.797 & 0.018 \\
3.000 & 1.150 & 0.026 \\
3.500 & 1.571 & 0.035 \\
4.000 & 2.061 & 0.047 \\
4.500 & 2.620 & 0.060 \\
5.000 & 3.251 & 0.074 \\
5.500 & 3.958 & 0.091 \\
6.000 & 4.741 & 0.110 \\
6.500 & 5.605 & 0.131 \\
7.000 & 6.554 & 0.154 \\
7.500 & 7.592 & 0.180 \\
8.000 & 8.725 & 0.208 \\
8.500 & 9.959 & 0.240 \\
9.000 & 11.302 & 0.276 \\
9.420 & 12.522 & 0.309 \\
\hline & &
\end{tabular}




\section{Conclusion}

In this work, we have studied the KL instability in rotating Rayleigh-Bénard convection with the free-free boundary conditions in the presence of the helical force. We modeled the system by a four-mode Lorenz model and extended it to include the KL instability.

We observed that for $S<3 \pi$, the critical Taylor number and critical angle for the onset of KL instability decrease as $S$ increases (column I). Then, this effect of helical force is similar to the modulation effect obtained by J. K. Bhattacharjee in rotating fluid layer under periodic modulation of the rotation rate (Bhattacharjee, 1990).

In the range $3 \pi \leq S \leq 14.0246$, the system exhibits the reentrant behavior of rolls demonstrating the constructive and destructive role of rotation. This behavior of reentrance observed with rolls is similar to that of hexagonal cells in convection for Boussinesq (Assenheimer \& Steinberg, 1996; Clever \& Busse, 1996) and non Boussinesq (Roy \& Steinberg, 2002; Madruga et al., 2006) approximations. We notice that in this domain, the $\mathrm{KL}$ instability exists for small values of Taylor number.

Another observation is that the presence of helical force in rotating Rayleigh-Bénard convection stabilizes the rolls against KL instability when its intensity exceeds the critical value 14.9246 (Column III).

\section{Acknowledgements}

We are grateful to Professor Joseph J. Niemela, head of ICTP fluid dynamics research group for his valuable suggestions. We would like to thank the Abdus Salam International Centre for Theoretical Physics (ICTP) under the Sandwich Training Educational Program (STEP) and the Academy of Sciences for Developing World (TWAS) for financial support.

\section{References}

Assenheimer, M., \& Steinberg, V. (1996). Observations of coexisting upflow and downflow hexagons in Boussinesq Rayleigh-Bénard convection. Phys. Rev. Lett., 76, 756-759. http://dx.doi.org/10.1103/PhysRevLett.76.756

Bhattacharjee, J. K., \& Mckane, A. J. (1988). Lorenz model for the rotating Rayleigh-Bénard convection problem. J. Phys. A: Math. Gen., 21, L555-L558. http://dx.doi.org/10.1088/0305-4470/21/10/004

Bhattacharjee, J. K. (1990). Convective instability in a rotating fluid layer under modulation of the rotation rate. Phys. Rev. A, 41, 5491-5494. http://dx.doi.org/10.1103/PhysRevA.41.5491

Busse, F. H., \& Heikes, K. E. (1980). Convection in a rotating layer: A simple case of turbulence. Science, 208, 173-175. http://dx.doi.org/10.1126/science.208.4440.173

Clever, R. M., \& Busse, F. H. (1996). Hexagonal convection cells under conditions of vertical symmetry. Phys. Rev. E, 53, R2037-R2040. http://dx.doi.org/10.1103/PhysRevE.53.R2037

Clune, T., \& Knobloch, E. (1993). Pattern selection in rotating convection with experimental boundary conditions. Phys. Rev. E, 47, 2536-2550. http://dx.doi.org/10.1103/PhysRevE.47.2536

Cox, S. M., \& Mattews P. C. (2000). Instability of rotating convection. J. Fluid Mech., 403, 153-172. http://dx.doi.org/10.1017/S0022112099006941

Essoun, M., \& Chabi Orou, J. B. (2010). Effects of rotation and helical force on the onset of Rayleigh-Bénard convection with free-free boundaries. African Physical Review 4(9), 65-71.

Küppers, G., \& Lortz, D. (1969). Transition from laminar convection to thermal turbulence in a rotating fluid layer. J. Fluid Mech., 35, 609-620. http://dx.doi.org/10.1017/S0022112069001327

Kurgansky, M. V. (1998). Vorticity generation in a moist atmosphere. Atmos. Oceanic Phys., 34(2), 156-161.

Levina, G. V., Moiseev, S. S., \& Rutkevich, P. B. (2000). Hydrodynamics alpha-effect in a Convective System, Advances in Fluid Mechanics, Nonlinear instability, Chaos and Turbulence. Eds. Debnath L. and Riahi D. N., Southampton: WIT. Press, 2, 111-162.

Levina, G. V., \& Burylov, I. A. (2006). Numerical simulation of helical-Vortex effects in Rayleigh-Bénard convection. Nonlinear Processes Geophysics, 13, 205-222. http://dx.doi.org/10.5194/npg-13-205-2006

Levina, G. V. (2006). Parameterization of Helical Turbulence in Numerical Models of Intense Atmospheric Vortices. Doklady Earth Sciences, 411A(9), 1417-1420. http://dx.doi.org/10.1134/S1028334X06090182

Liu, J., \& Ahlers G. (1997). Rayleigh-Bénard convection in binary-gas mixtures: Thermophysical properties and the onset of convection. Phys. Rev. E, 55, 6950-6968. http://dx.doi.org/10.1103/PhysRevE.55.6950 
Lorenz, E. N. (1963). Deterministic Nonperiodic flow. J. Atmos. Sci., 20, 130-141. http://dx.doi.org/10.1175/1520-0469(1963)020<0130:DNF>2.0.CO;2

Madruga, S., Riecke, H., \& Pesch, W. (2006). Reentrant hexagons in non-Boussinesq convection. J. Fluid Mech., 548, 341-360. http://dx.doi.org/10.1017/S0022112005007640

Moiseev, S. S., Sagdeev, R. Z., Tur, A. V., Khomenho, G. A., \& Yanovsky, V. V. (1983) . Theory of development of large-scale structures in hydrodynamic turbulence. Sov. Phys. JETP, 58, 1149-1157.

Moiseev, S. S., Rutkevich, P. B., Tur, A. V., \& Yanovsky, V. V. (1988). Vortex dynamo in a Convective medium with helical turbulence. Sov. Phys. JETP, 67(2), 294-299.

Murali, P., Tagare, S. G., \& Hanumantha Ravi, P. V. (1994). Küppers-Lortz instability in a rapidly rotating inviscid magnetoconvection. Phys. Rev. E, 50, 1665-1669. http://dx.doi.org/10.1103/PhysRevE.50.1665

Niemela, J. J., \& Donnelly, R. J. (1986). Direct transition to turbulence in rotating Bénard convection, Phys. Rev. Lett., 57, 2524-2527. http://dx.doi.org/10.1103/PhysRevLett.57.2524

Ponty, Y., Passot, T., \& Sulem P. L. (1997). A new instability for finite Prandtl number rotating convection with free- slip boundary conditions. Phys. Fluids, 9, 67-75. http://dx.doi.org/10.1063/1.869176

Roy, A., \& Steinberg, V. (2002). Reentrant hexagons in non-Boussinesq convection: Effect of compressibility. Phys. Rev. Lett., 88, 244503-1--244503-4. http://dx.doi.org/10.1103/PhysRevLett.88.244503

Rutkevich, P. B. (1993). Equation for the rotational instability due to convective turbulence and the Coriolis force. JETP, 77(6), 933-938.

Sparrow, C. (1982). The Lorenz equations: Bifurcations, Chaos and Strange Attractors. Applied Mathematics Sciences, 41. New York: Springer-Verlag. http://dx.doi.org/10.1007/978-1-4612-5767-7

Steenbeck, M., Krause, F., \& Rädler, K. H. (1966). Zur Berechnung der mittleren Lorentz-Feldstärke $\vec{v} \times \vec{B}$ fur ein elektrisch leitendes Medium in turbulenter, durch Coriolis-Kräfte beeinflusster Bewegung. $Z$. Naturforsch, 21a, 369-376.

Stein, N. D. (1989). Oscillator convection and chaos in a Lorenz-type model of rotating fluid. J. Stat. Phys., 56, 841-877. http://dx.doi.org/10.1007/BF01016782

Tritton, D. J. (1988). Physical Fluid Dynamics (2nd Ed.). Oxford Science Publications. 